\title{
Fibroblast growth factor 21 predicts outcome in community-acquired pneumonia: secondary analysis of two randomised controlled trials
}

\author{
Fahim Ebrahimi $\mathbb{1}^{1}$, Carole Wolffenbuttel ${ }^{2}$, Claudine A. Blum $^{1,3}$, \\ Christine Baumgartner ${ }^{4}$, Beat Mueller ${ }^{3}$, Philipp Schuetz ${ }^{3}$, Christian Meier ${ }^{1,5}$, \\ Marius Kraenzlin ${ }^{1,5}$, Mirjam Christ-Crain ${ }^{1}$ and Matthias Johannes Betz ${ }^{1}$
}

Affiliations: ${ }^{1}$ Dept of Endocrinology, Diabetes and Metabolism, University Hospital Basel, Basel, Switzerland. ${ }^{2}$ Dept of Internal Medicine, Utrecht University, Utrecht, The Netherlands. ${ }^{3}$ University Dept of Medicine, Kantonsspital Aarau, Aarau, Switzerland. ${ }^{4}$ Dept of General Internal Medicine, Inselspital, Bern University Hospital, Bern, Switzerland. ${ }^{5}$ Medical Faculty, University of Basel, Basel, Switzerland.

Correspondence: Matthias J. Betz, Dept of Endocrinology, Diabetes and Metabolism, University Hospital Basel, Petersgraben 4, 4031 Basel, Switzerland. E-mail: matthias.betzQusb.ch

@ERSpublications

Fibroblast growth factor 21 predicts higher risk for clinical instability and 30-day mortality in moderate-to-severe community-acquired pneumonia http://ow.ly/8LVe30mWrjH

Cite this article as: Ebrahimi F, Wolffenbuttel C, Blum CA, et al. Fibroblast growth factor 21 predicts outcome in community-acquired pneumonia: secondary analysis of two randomised controlled trials. Eur Respir J 2019; 53: 1800973 [https://doi.org/10.1183/13993003.00973-2018].

ABSTRACT Acute systemic inflammatory conditions are accompanied by profound alterations of metabolism. However, the role of fibroblast growth factor 21 (FGF21), a recently identified central regulator of metabolism, is largely unknown in community-acquired pneumonia (CAP). This study aims to characterise the pattern of FGF21 in pneumonia and associations with disease severity and outcome.

This is a secondary analysis of two independent multicentre randomised controlled trials in patients presenting to the emergency department with CAP. Primary and secondary efficacy parameters included 30-day mortality, length of hospital stay, time to clinical stability and duration of antibiotic treatment.

A total of 509 patients were included in the analysis. FGF21 levels at admission strongly correlated with disease severity, as measured by the Pneumonia Severity Index. Increased levels of FGF21 were associated with prolonged time to clinical stability, antibiotic treatment and hospitalisation. FGF21 levels at admission were significantly higher in nonsurvivors than in survivors, yielding a 1.61-fold increased adjusted odds ratio of 30-day mortality (95\% CI 1.21-2.14; p=0.001). Moreover, FGF21 was found to identify patients for 30-day mortality with superior discriminative power compared with routine diagnostic markers.

Moderate-to-severe CAP patients with higher levels of FGF21 were at increased risk for clinical instability, prolonged hospitalisation and 30-day all-cause mortality.

This article has supplementary material available from erj.ersjournals.com

The two trials analysed in this study are registered at ClinicalTrials.gov with identifier number NCT00973154 (STEP) and at the ISRCTN Registry with identifier ISRCTN04176397 (ProCAP). Individual participant data that underlie the results reported in this article, after de-identification (text, tables, figures and appendices, study protocols) will be available to investigators whose proposed use of the data has been approved by an independent review committee ("learned intermediary") identified for the purpose of an individual participant data meta-analysis, beginning 9 months and ending 36 months after article publication. Proposals should be directed to the corresponding author. To gain access, data requestors will need to sign a data access agreement. 


\section{Introduction}

Community-acquired pneumonia (CAP) is a major cause of morbidity, mortality and related socioeconomic costs worldwide [1, 2]. CAP-related mortality remains high despite recent advances in medical care. The World Health Organization estimated in 2016 that lower respiratory tract infections were the fourth leading cause of death globally and the number one cause of death in low-income countries [3]. Clinical signs and symptoms (e.g. fever and chills) or routine laboratory parameters (e.g. C-reactive protein (CRP) or white blood cell (WBC) count) have been shown to be of only limited value for the management and prognosis of severe lower respiratory tract infections $[4,5]$. Hence, prognostic scoring rules have been developed with the aim to integrate the complex pathophysiological changes in order to predict severity and outcome of CAP and to guide therapy. Of these, the Pneumonia Severity Index (PSI) and CURB-65 (confusion, urea $>7 \mathrm{mmol} \cdot \mathrm{L}^{-1}$, respiratory rate $\geqslant 30$ breaths $\cdot \mathrm{min}^{-1}$, blood pressure $<90 \mathrm{mmHg}$ (systolic) or $\leqslant 60 \mathrm{mmHg}$ (diastolic), age $\geqslant 65$ years) are the most common prognostic classification scores [6]. However, calculating these scores has been shown to be reasonably complex and time consuming, which leads to the fact that they are often not used and that the majority of clinicians are not able to accurately calculate the scores [7]. Against this background a simple and reliable approach is needed to help identify patients at risk and streamline management decisions in patients presenting with severe CAP.

Fibroblast growth factor 21 (FGF21) has recently been identified as a central regulator of metabolism via adaptation of glucose homeostasis, ketogenesis, insulin sensitivity and lipid metabolism [8, 9]. Furthermore, it probably plays a major role in the regulation of energy expenditure, enhancing substrate utilisation and potently inducing thermogenesis in brown adipose tissue [10-13]. Several studies have suggested the potential role of FGF21 in the maintenance and regulation of energy homeostasis and mitochondrial function in conditions of metabolic stress [14, 15]. The capacity of FGF21 to facilitate thermogenesis in brown adipose tissue suggests that it might also be involved in the elevation of body temperature in systemic inflammatory states.

However, while acute systemic inflammatory conditions are accompanied by profound alterations of metabolism such as hyperglycemia, insulin resistance and mitochondrial dysfunction [16-18], the role of the central regulator FGF21 is still unknown. Emerging data suggest that FGF21 is a positive acute-phase protein since systemic inflammation has been shown to induce $\sim 2$-fold increase in serum FGF21, which might contribute to adipose tissue lipolysis and ketone production that occur during inflammation $[19,20]$. In addition, studies have shown that FGF21 potentially plays an anti-inflammatory and immunoregulatory role by downregulation of the pro-inflammatory cytokine interleukin (IL)- $1 \beta$ and upregulation of the anti-inflammatory cytokine IL-10 [20]. FGF21 might therefore protect from overwhelming systemic inflammation in severe inflammatory states such as lower respiratory tract infections.

In this study we investigated the potential role of FGF21 as a predictive biomarker in CAP, using samples and clinical data from two large, well-defined patient cohorts with moderate-to-severe lower respiratory tract infections (ProCAP (Procalcitonin (PCT)-guided reduction of the duration of antibiotic therapy in CAP; ISRCTN Registry identifier ISRCTN04176397) [21] and STEP (Corticosteroid treatment for CAP; ClinicalTrials.gov identifier NCT00973154) [22]). The main aims of this study were to assess and confirm the clinical use of FGF21 for early diagnosis and clinical guidance of therapy, to predict adverse outcome, and to evaluate its interaction with adjunct corticosteroid treatment in CAP.

\section{Materials and methods \\ Study subjects and design}

This is a secondary analysis of two investigator-initiated, parallel-group, randomised, multicentre trials involving patients with all severities of CAP admitted to the emergency department. Full details of the trial designs and results have been published [21-23]. In brief, consecutive patients (aged $\geqslant 18$ years) presenting with CAP were enrolled at emergency departments or medical wards of tertiary care hospitals in Switzerland within $24 \mathrm{~h}$ of presentation.

Patients in the ProCAP trial were randomised (1:1 ratio) to either PCT-guided antibiotic treatment or usual care [21]. In the STEP trial, patients were randomly assigned (1:1 ratio) to receive either $50 \mathrm{mg}$ prednisone or placebo daily for 7 days as adjunct treatment. Patients, treating physicians, investigators and data assessors were masked to treatment allocation [22].

The conduct of the trials adhered to the Declaration of Helsinki and Good Clinical Practice guidelines, and ethical committees of all participating hospitals approved the studies before patient recruitment.

\section{Methods}

Informed consent was obtained within $24 \mathrm{~h}$ of admission to hospital. All patients were treated according to international CAP consensus guidelines [24]. Baseline data included medical history, relevant 
comorbidities, clinical variables relating to pneumonia and all variables required for the calculation of the PSI [25]. Clinical study data were systematically collected to obtain 30-day outcome after admission.

Main outcome parameters included time to effective hospital discharge (length of stay), 30-day all-cause mortality, duration of intravenous and overall antibiotic treatment, time to clinical stability (TTCS; defined as the time to stabilisation of vital signs at two consecutive measurements $\geqslant 12 \mathrm{~h}$ apart [22]), admission to the intensive care unit (ICU), and CAP complications (including recurrence, acute respiratory distress syndrome, empyema, nosocomial infections until day 30 and severe adverse events possibly related to CAP, ICU admission or readmission to hospital).

\section{Analysis of FGF21}

Blood samples from each patient were collected upon emergency department admission and on day 3 , and were subsequently stored at $-80^{\circ} \mathrm{C}$. For this analysis, remaining serum samples from the ProCAP trial were still available for 150 patients. In the STEP trial, all patients from the main study site (University Hospital Basel ( $n=359$ : 177 patients allocated to placebo and 182 patients allocated to prednisone)) were included. FGF21 was measured in serum samples using the human FGF21 Quantikine immunoassay (R\&D Systems, Minneapolis, MN, USA) for ProCAP samples and the human FGF21 Ella Simple Plex assay (ProteinSimple, San Jose, CA, USA) for STEP samples. Both assays are based on an identical monoclonal antibody specific for human FGF21, which detects total FGF21 independent of plasmatic proteolytic N- or C-terminal cleavage [26]. The two assays correlate well $\left(\mathrm{R}^{2}=0.97\right.$; information provided by R\&D/ProteinSimple). The Quantikine ELISA has a limit of detection for human FGF21 of $8.69 \mathrm{pg} \cdot \mathrm{mL}^{-1}$ and an inter-assay coefficient of variation of $5.2-10.9 \%$. The Simple Plex assay has a limit of detection of $3.74 \mathrm{pg} \cdot \mathrm{mL}^{-1}$ and an inter-assay coefficient of variation of $7.8 \%$; mean values of three independent measurements were taken for analysis. Reference values for young healthy subjects were measured in 56 participants from the FluvaBAT and MIBAT studies (ClinicalTrials.gov identifiers NCT03189511 and NCT02682706, respectively). Reference values for elderly healthy subjects were measured in serum samples from 40 patients from an endocrine outpatient clinic in Basel, Switzerland. All healthy participants gave written informed consent.

\section{Statistical analysis}

Unless stated otherwise, categorical variables are expressed as number (percentage) and continuous variables as median (interquartile range (IQR)). The distribution of FGF21 was right-skewed. After $\log _{10}$ transformation, the distribution approximated a normal distribution.

Univariate and multivariate regression models were used for analyses of associations of comorbidities with baseline levels of FGF21. Unadjusted and adjusted estimates of the effect size and corresponding 95\% confidence intervals were determined using linear, logistic or Cox proportional hazards regression as appropriate. All multivariate models were adjusted for the same variables: patient age, sex, diabetes mellitus, congestive heart failure (CHF) and renal insufficiency. Analyses on STEP study participants were additionally adjusted for randomised treatment. Kaplan-Meier curves were used to illustrate TTCS and length of hospital stay based on FGF21 tertiles (highest tertile versus lower two tertiles). Log-rank tests were used to detect differences between groups. Receiver operating characteristic (ROC) analyses were performed to analyse the discriminative power to identify patients at risk for 30-day mortality. Correlation analyses were performed calculating Spearman's rho (r). For analyses, consistent clinical and laboratory variables of the ProCAP and STEP trials were pooled. All statistical analyses were performed using Stata version 14.2 (StataCorp, College Station, TX, USA) and tests were done at a two-sided 5\% significance level with two-sided $95 \%$ confidence intervals.

\section{Results}

\section{Patient characteristics}

In total, 509 patients were included in this study: 150 from the ProCAP trial population and 359 from the STEP trial population (baseline characteristics of patients are presented in tables 1 and 2, respectively; a combined table of baseline characteristics is included in supplementary table S1). ProCAP patients were (median) 73 years old and 62.0\% were male; STEP patients were (median) 75 years old and 63.2\% were male. The burden of pre-existing pulmonary disease was relatively low in both studies, with a history of chronic obstructive pulmonary disease (COPD) in $21.3 \%$ and $18.9 \%$ in ProCAP and STEP, respectively. The prevalence of diabetes mellitus type 2 was $23.3 \%$ and $22.8 \%$ in ProCAP and STEP, respectively. Significantly fewer patients had pre-existing CHF in ProCAP (6.0\%) than in STEP (20.6\%). Overall, more than half of the patients $(57.3 \%$ and $54.6 \%$ in ProCAP and STEP, respectively) had severe pneumonia classified in the high-risk PSI classes IV and V. 
Association of FGF21 with pneumonia severity

At baseline, median (IQR) serum FGF21 levels were highly increased (456.5 (181.2-1127.9) pg.mL $\mathrm{m}^{-1}$ ) compared with values in a population of elderly healthy controls $\left(140.2(81.1-161.8) \mathrm{pg} \cdot \mathrm{mL}^{-1} ; \mathrm{p}<0.001\right)$ and almost 10 -fold higher than in young healthy controls $\left(50.4(13.7-113.3) \mathrm{pg} \cdot \mathrm{mL}^{-1} ; \mathrm{p}<0.001\right)$. They subsided until day 3 when they were still 2 -fold elevated $\left(299.9(157.9-659.9) \mathrm{pg} \cdot \mathrm{mL}^{-1}\right)$ when compared with elderly healthy controls (characteristics of healthy controls are shown in supplementary table S2). We found a significant positive correlation of FGF21 levels at emergency admission with PCT levels ( $\mathrm{r}=0.186$, $\left.\mathrm{R}^{2}=0.045 ; \mathrm{p}<0.001\right)$, but not with CRP levels $\left(\mathrm{r}=-0.058, \mathrm{R}^{2}=0.001 ; \mathrm{p}=0.19\right)$ (supplementary figures $\mathrm{S} 3$ and S4). Likewise, there was no correlation of FGF21 levels with body temperature (in-ear) at emergency admission (supplementary figure S5).

On admission, levels of FGF21 increased stepwise with higher PSI classes of CAP severity (figure 1a and c for each study separately; supplementary figure S1 for the pooled cohort). There was a linear correlation of $\log _{10}$-transformed FGF21 with the PSI score both in the ProCAP cohort $\left(\mathrm{R}^{2}=0.1667 ; \mathrm{p}<0.001\right)$ as well as in the STEP cohort $\left(\mathrm{R}^{2}=0.1604 ; \mathrm{p}<0.001\right)$ (supplementary figure S6). Patients with severe pneumonia categorised as PSI classes IV or V had markedly higher median (IQR) baseline levels of FGF21 (717.3 (291.5-1530.1) pg. $\mathrm{mL}^{-1}$ ) than patients with mild-to-moderate severity categorised as PSI classes I, II or III (270.5 (123.3-626.9) pg.mL $\left.\mathrm{mL}^{-1} ; \mathrm{p}<0.001\right)$ (supplementary figure S2). In the ROC analyses, FGF21 significantly better discriminated patients with high risk (PSI IV and V) from those with low risk (PSI I, II and III) when compared with established biomarkers. The area under the curve (AUC) for FGF21 was 0.68 (95\% CI 0.64-0.73), for CRP 0.48 (95\% CI 0.43-0.53; p<0.001), for PCT 0.56 (95\% CI $0.51-0.62$; $\mathrm{p}=0.02)$ and for WBC count 0.46 (95\% CI $0.41-0.51 ; \mathrm{p}<0.001)$ (supplementary table S4).

\section{TABLE 1 Baseline characteristics and clinical variables of enrolled patients: ProCAP trial}

\begin{tabular}{|c|c|}
\hline Subjects & 150 \\
\hline \multicolumn{2}{|l|}{ General characteristics } \\
\hline Age years & $73.0(56.0-82.0)$ \\
\hline Male & 93 (62.0) \\
\hline $\mathrm{BMI} \mathrm{kg} \cdot \mathrm{m}^{-2}$ & $24.8(22.2-28.7)$ \\
\hline Smoker & 32 (21.3) \\
\hline Pack-years & $30.0(20.0-50.0)$ \\
\hline \multicolumn{2}{|l|}{ Comorbidities } \\
\hline Diabetes mellitus & $35(23.3)$ \\
\hline COPD & 32 (21.3) \\
\hline Asthma & $3(2.0)$ \\
\hline Heart failure & $9(6.0)$ \\
\hline Coronary artery disease & 47 (31.3) \\
\hline Cerebrovascular disease & $9(6.0)$ \\
\hline Renal insufficiency & $34(22.7)$ \\
\hline Neoplastic disease & 23 (15.3) \\
\hline Antibiotic pre-treatment & $31(20.7)$ \\
\hline \multicolumn{2}{|l|}{ Clinical variables } \\
\hline Systolic blood pressure mmHg & $130.0(113.0-142.0)$ \\
\hline Heart rate beats $\cdot \min ^{-1}$ & $96.0(84.0-108.0)$ \\
\hline Respiratory rate breaths $\cdot \min ^{-1}$ & $22.0(18.0-27.0)$ \\
\hline Body temperature (in-ear) ${ }^{\circ} \mathrm{C}$ & 38.5 (37.7-39.3) \\
\hline SIRS points & $3(2-4)$ \\
\hline \multicolumn{2}{|l|}{ PSI class ${ }^{\#}$} \\
\hline I, II and III & $64(42.7)$ \\
\hline IV and V & 86 (57.3) \\
\hline PSI score ${ }^{\#}$ & $95(71-115)$ \\
\hline \multicolumn{2}{|l|}{ Laboratory values } \\
\hline $\mathrm{C}$-reactive protein $\mathrm{mg} \cdot \mathrm{L}^{-1}$ & $135.5(74.0-216.4)$ \\
\hline Procalcitonin $\mathrm{ng} \cdot \mathrm{dL}^{-1}$ & $0.4(0.2-1.4)$ \\
\hline White blood cell count $\times 10^{9} \mathrm{~L}^{-1}$ & $12.8(9.0-15.4)$ \\
\hline Fasting glucose $\mathrm{mmol} \cdot \mathrm{L}^{-1}$ & $6.8(5.8-8.7)$ \\
\hline
\end{tabular}

Data are presented as $n$, median (IQR) or $n(\%)$. BMI: body mass index; COPD: chronic obstructive pulmonary disease; SIRS: systemic inflammatory response syndrome; PSI: Pneumonia Severity Index. \#: the PSI is a clinical prediction rule to calculate the probability of morbidity and mortality in patients with community-acquired pneumonia [25]; PSI risk class I corresponds to age $\leqslant 50$ years and no risk factors ( $\leqslant 50$ points), risk class II to $<70$ points, risk class III to 71-90 points, risk class IV to 91-130 points and risk class $V$ to $>130$ points. 
Patients in the highest tertile of FGF21 levels $\left(>833.3 \mathrm{pg} \cdot \mathrm{mL}^{-1}\right)$ required 2 days longer to achieve clinical stability than patients in the lower two tertiles (median (IQR) 6.0 (3.0-10.0) versus 4.0 (2.0-7.4) days). The adjusted hazard ratio for Cox regression of FGF21 at baseline was 0.87 (95\% CI 0.80-0.95; p=0.002); a HR $<1.0$ corresponding to prolonged TTCS (figure 2a and table 3; data shown for each study separately in supplementary tables S5 and S6). Accordingly, higher FGF21 was associated with longer duration of i.v. and total antibiotic treatment for each stepwise increase in $\log _{10}$-transformed FGF21 (table 3 ).

\section{FGF21 and clinical outcome}

Median (IQR) length of hospital stay was significantly longer in patients in the highest FGF21 tertile (10.0 (7.0-16.0) days) compared with the lower two tertiles (8.0 (5.0-14.0) days), resulting in an adjusted HR of 0.93 (95\% CI $0.87-0.99 ; \mathrm{p}=0.03$ ) (figure $2 \mathrm{~b}$ and table 3 ; data shown for each study separately in supplementary tables S5 and S6). Overall, complications associated with CAP (i.e. acute respiratory distress syndrome, empyema, respiratory failure with intubation, persistence of pneumonia and CAP-associated mortality) tended to be higher in the third tertile of FGF21 compared with the lower two tertiles (44.5\% versus $30.4 \%)$; however, the adjusted odds ratio did not meet statistical significance $(\mathrm{p}=0.15)$. The rate of ICU admissions was increased ( $13.6 \%$ in the third tertile versus $8.5 \%$ in the first two tertiles). For each

TABLE 2 Baseline characteristics and clinical variables of enrolled patients: STEP trial

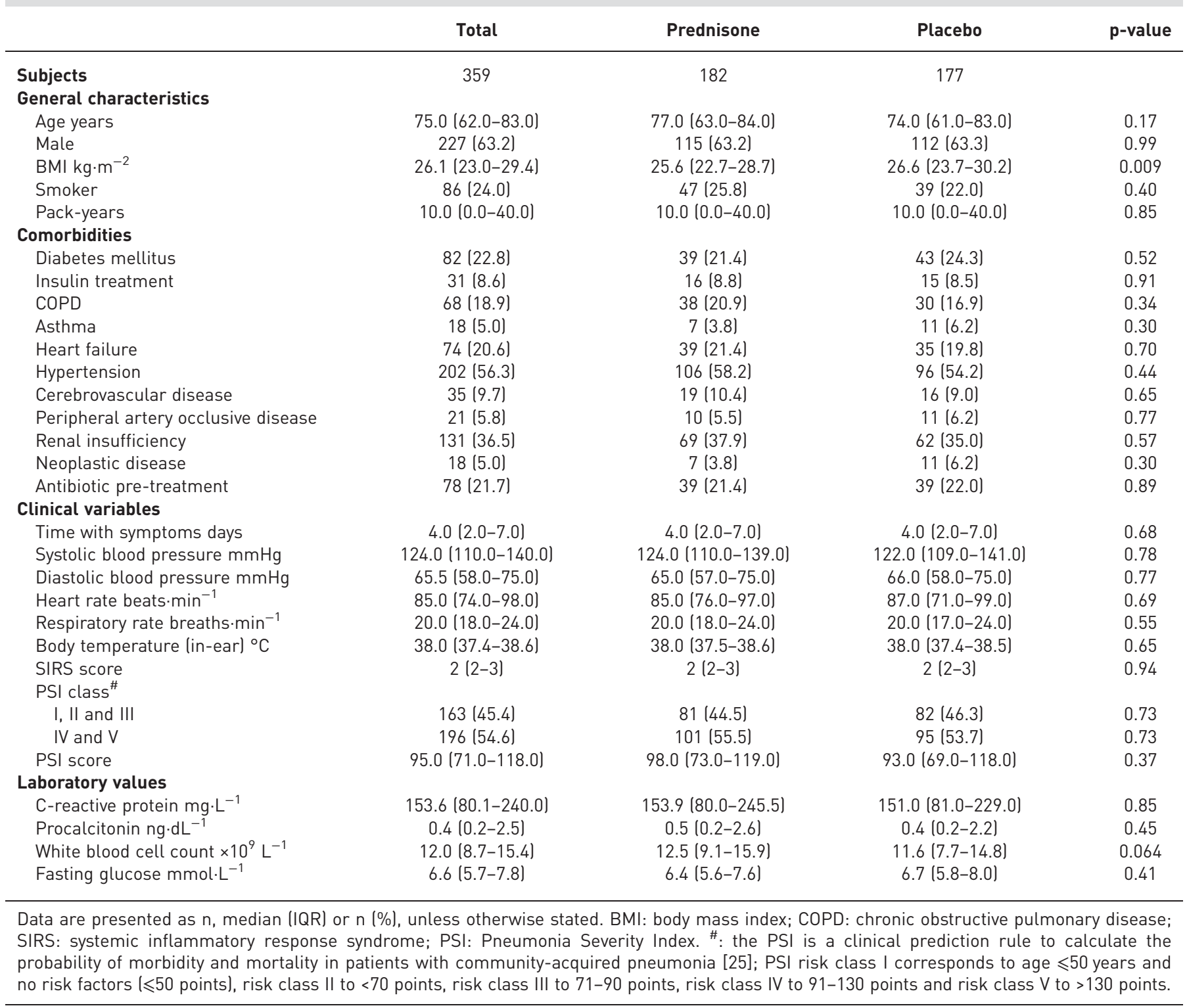



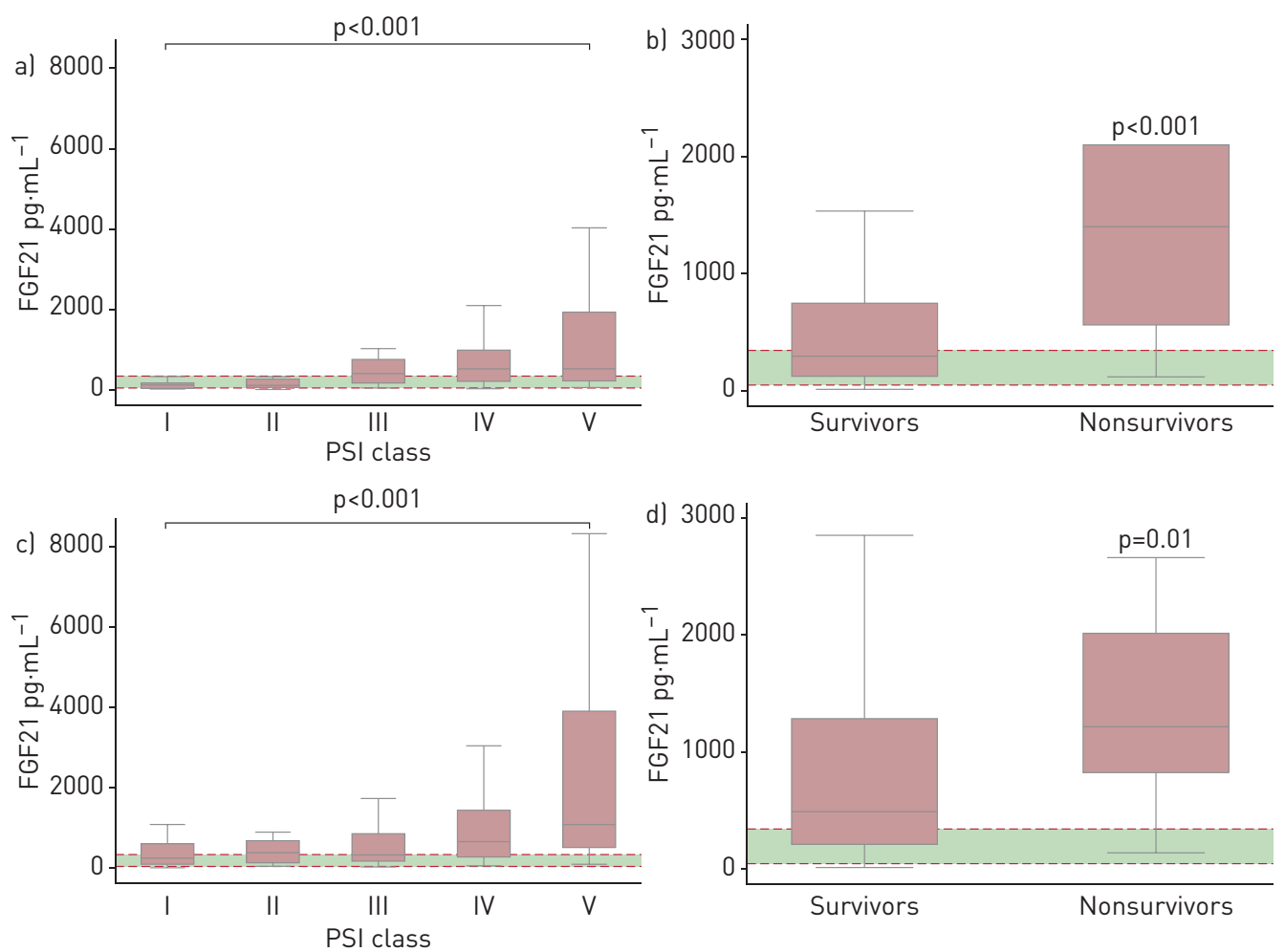

FIGURE 1 Box plots for levels of fibroblast growth factor 21 (FGF21) at baseline stratified a, c) by Pneumonia Severity Index (PSI) class or $b, d$ ) by survivors versus nonsurvivors for each trial separately: $a, b$ ) ProCAP trial and $c$, d) STEP trial. Each box signifies the upper and lower quartiles; the median is represented by a line within the box. Whiskers represent the upper and lower adjacent values; outliers are not depicted. The horizontal dashed red lines and green area represent the $95 \%$ confidence interval of elderly healthy controls (5th percentile $47.5 \mathrm{pg} \cdot \mathrm{mL}^{-1}$; 95 th percentile $343.8 \mathrm{pg} \cdot \mathrm{mL}^{-1}$ ). FGF21 discriminates between PSI classes I, II and III versus classes IV and V, as the high-risk classes have FGF21 levels clearly above the $95 \%$ confidence intervals of age-matched controls (a and c). FGF21 levels at baseline potently separated nonsurvivors from survivors ( $b$ and $d$ ).

increment in baseline $\log _{10}$-transformed FGF21 levels the adjusted OR was 1.32 (95\% CI 1.07-1.65; $\mathrm{p}=0.01$ ) (table 3).

\section{FGF21 and CAP-associated mortality}

Levels of FGF21 were significantly higher in nonsurvivors than in corresponding survivors (1307.6 versus $416.7 \mathrm{pg} \cdot \mathrm{mL}^{-1} ; \mathrm{p}<0.001$ ) (figure $1 \mathrm{~b}$ ). In fully adjusted logistic regression analyses, admission levels of FGF21 were associated with an adjusted OR of 1.61 for 30-day mortality for each stepwise increase in $\log _{10}$-transformed FGF21 (95\% CI 1.21-2.14; p=0.001) (table 3).

When compared with PCT and CRP, FGF21 was superior for the identification of patients at risk for 30-day mortality as assessed by comparisons of AUC of the related ROC curves: baseline FGF21 levels had an AUC of 0.73 (95\% CI 0.65-0.82), which was significantly superior to CRP (AUC 0.47, 95\% CI 0.37 $0.58 ; \mathrm{p}<0.001$ ), PCT (AUC 0.62, 95\% CI 0.53-0.71; $\mathrm{p}=0.03$ ) and WBC count (AUC 0.31, 95\% CI $0.22-$ $0.39 ; \mathrm{p}<0.001$ ) (figure 3 and table 4 ).

When compared with prognostic scoring scales, baseline FGF21 levels performed better than the CURB-65 score and comparable to the PSI score (table 4 and figure 3). Accordingly, of the 32 patients who died within 30 days only two patients had an admission FGF21 level below the 95th percentile derived from the elderly healthy reference population. Both patients were not admitted to the ICU and had PSI scores of 93 and 94 points, which were just above the lower limit of 91 points to be classified into PSI class IV. In comparison, of the 32 patients who died, one patient was misclassified by the PSI into the low-to-intermediate risk classes I-III. This patient had a baseline FGF21 of $369.7 \mathrm{pg} \cdot \mathrm{mL}^{-1}$ and would have been correctly categorised as high risk by the admission FGF21 level.

When clinical outcomes were analysed for each trial population separately, the association with mortality was still distinctive despite reduced statistical power (supplementary tables S5 and S6). 


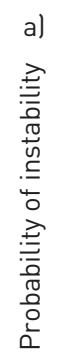

\section{At risk $\mathrm{n}$}

FGF21 T1-T2 240

FGF21 T3 119

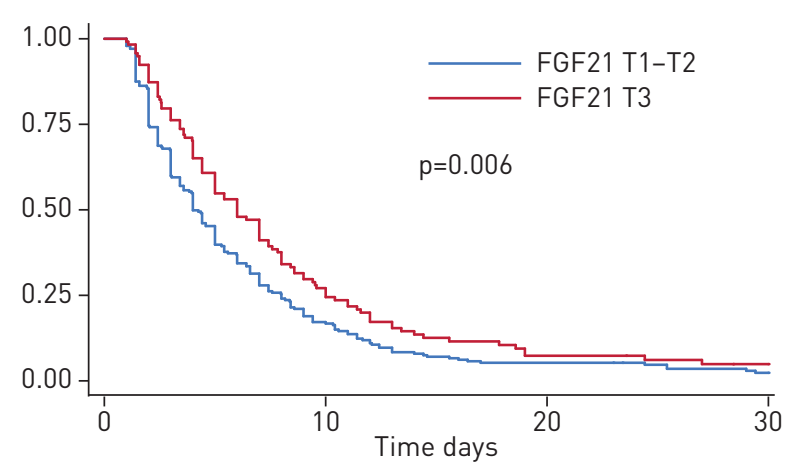

$40 \quad 11$

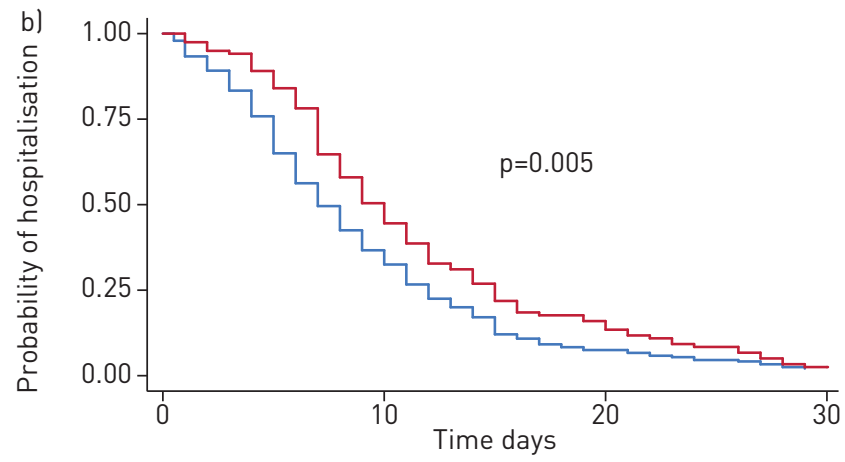

At risk $n$

4 FGF21 T1-T2 240

3 FGF21 T3 119
88

60
18

19

FIGURE 2 Kaplan-Meier estimators of a) time to clinical stability (TTCS) and b) length of hospital stay for the pooled cohort according to initial

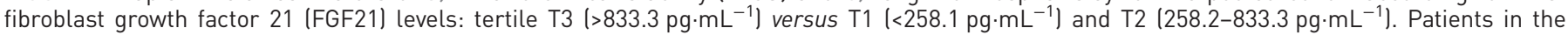
third tertile of FGF21 levels had significantly prolonged TTCS and length of hospital stay. p-values determined by log-rank test.

\section{Associations of baseline FGF21 levels with demographic characteristics, comorbidities and clinical variables}

Multivariate linear regression models were used to investigate predictors of increased levels of FGF21 at emergency admission. In univariate analysis, age was associated with increased levels of FGF21; however, after multivariable adjustment this association was no longer significant $(\mathrm{p}=0.59)$ (table 5). While patients with a history of CHF (adjusted difference $226.9 \mathrm{pg} \cdot \mathrm{mL}^{-1}, 95 \%$ CI $13.7-440.1 \mathrm{pg} \cdot \mathrm{mL}^{-1}$; $\mathrm{p}=0.04$ ) and with chronic kidney disease (CKD) (adjusted difference $255.2 \mathrm{pg} \cdot \mathrm{mL}^{-1}, 95 \% \mathrm{CI} 72.0-438.4 \mathrm{pg} \cdot \mathrm{mL}^{-1}$; $\mathrm{p}=0.006$ ) had significantly higher levels of FGF21 at baseline, male sex was associated with lower levels (adjusted difference $-238.4 \mathrm{pg} \cdot \mathrm{mL}^{-1}$, 95\% CI $-396.4--80.3 \mathrm{pg} \cdot \mathrm{mL}^{-1} ; \mathrm{p}=0.003$ ) (table 5).

\section{Effects of corticosteroid treatment on FGF21}

Patients in the STEP trial were randomly allocated to receive treatment with either $50 \mathrm{mg}$ prednisone or placebo daily for 7 days. Prednisone treatment was associated with a median (IQR) accelerated decline in FGF21 levels from $592.8(231.5-1336.0)$ to $250(134.6-489.5) \mathrm{pg} \cdot \mathrm{mL}^{-1}$ at day 3 compared with almost no change in the placebo group. Overall, there was a significant between-group difference of $-811.3 \mathrm{pg} \cdot \mathrm{mL}^{-1}$ (95\% CI $-1448.2--174.2 \mathrm{pg} \cdot \mathrm{mL}^{-1} ; \mathrm{p}=0.01$ ) (supplementary figure $\mathrm{S} 7$ ).

TABLE 3 Overview of fibroblast growth factor 21 (FGF21) and relevant clinical outcomes

Tertiles 1-2 Tertile 3

Regression analysis of baseline FGF21

\begin{tabular}{|c|c|c|c|}
\hline $\begin{array}{c}\text { Univariate regression } \\
\text { analysis OR, HR or regression } \\
\text { coefficient }(95 \% \mathrm{CI})\end{array}$ & p-value & $\begin{array}{l}\text { Multivariable adjusted } \\
\text { regression } \\
\text { analysis OR, HR or regression } \\
\text { coefficient }(95 \% \mathrm{CI})\end{array}$ & p-value \\
\hline
\end{tabular}

\begin{tabular}{|c|c|c|c|c|c|c|}
\hline Subjects & 340 & 169 & & & & \\
\hline Death (30 days) & $11(3.2)$ & $21(12.4)$ & $1.60(1.25-2.04)^{9}$ & $<0.001$ & $1.61(1.21-2.14)^{9}$ & 0.001 \\
\hline $\begin{array}{l}\text { Time to effective hospital } \\
\text { discharge days }\end{array}$ & $8.0(5.0-14.0)$ & $10.0(7.0-16.0)$ & $0.89(0.84-0.96)^{\#}$ & 0.001 & $0.93(0.87-0.99)^{\#}$ & 0.03 \\
\hline $\begin{array}{l}\text { Total duration of antibiotic } \\
\text { treatment days }\end{array}$ & $10.0(7.0-13.0)$ & $10.0(7.0-14.0)$ & $0.68(0.29-1.08)^{+}$ & 0.001 & $0.56(0.12-0.99)^{+}$ & 0.01 \\
\hline $\begin{array}{l}\text { Intravenous antibiotic } \\
\text { treatment days }\end{array}$ & $5.0(3.0-7.0)$ & $6.0(5.0-8.0)$ & $0.91(0.54-1.28)^{+}$ & $<0.001$ & $0.71(0.31-1.12)^{+}$ & 0.001 \\
\hline ICU admission & $29(8.5)$ & $23(13.6)$ & $1.36(1.12-1.67)^{9}$ & 0.002 & $1.32(1.07-1.65)^{9}$ & 0.01 \\
\hline TTCS $^{\S}$ days & $4.0(2.0-7.4)$ & $6.0(3.0-10.0)$ & $0.87(0.80-0.94)^{\#}$ & $<0.001$ & $0.87(0.80-0.95)^{\#}$ & 0.002 \\
\hline CAP complications $f$ & $73(30.4)$ & 53 (44.5) & $1.23(1.05-1.45)^{9}$ & 0.01 & $1.14(0.96-1.36)^{9}$ & 0.15 \\
\hline
\end{tabular}

Data are presented as $\mathrm{n}, \mathrm{n}(\%)$ or median (interquartile range), unless otherwise stated, and adjusted for age, sex, diabetes mellitus, congestive heart failure and renal insufficiency; FGF21 values were $\log _{10}$ transformed. ICU: intensive care unit; TTCS: time to clinical stability;

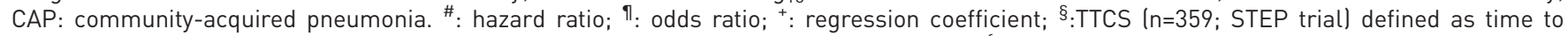
clinical stabilisation of vital signs at two consecutive measurements $\geqslant 12 \mathrm{~h}$ apart [22]; ${ }^{f}$ : CAP complications ( $\mathrm{n}=359$; $\mathrm{STEP}$ trial) defined as recurrence, acute respiratory distress syndrome, empyema, nosocomial infections until day 30 , or serious adverse events possibly related to CAP, ICU stay or readmission to hospital. 


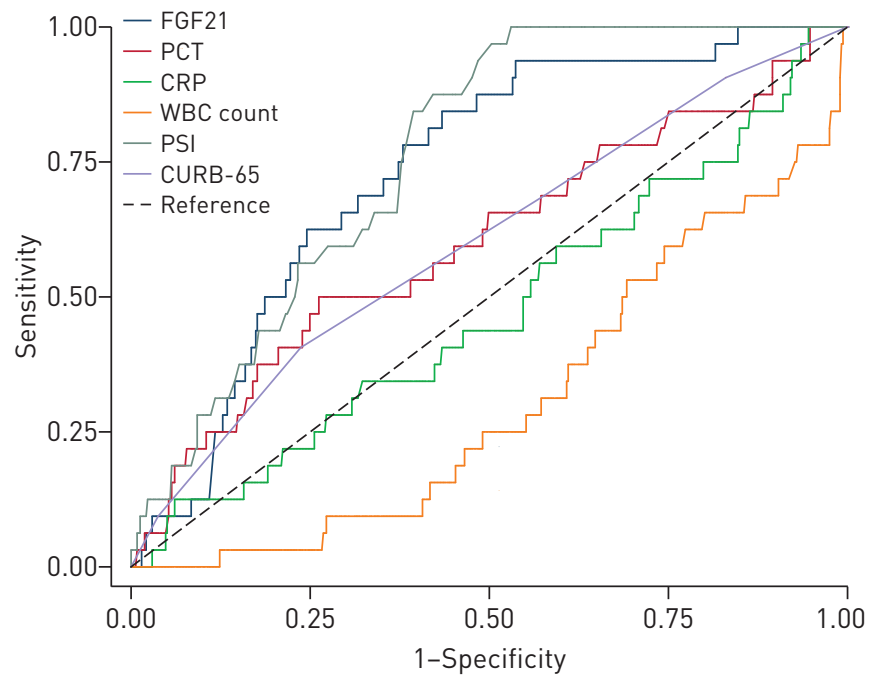

FIGURE 3 Receiver operating characteristic curve analysis of various laboratory parameters versus clinical parameters predicting survival from community-acquired pneumonia in the pooled cohort. FGF21: fibroblast growth factor 21; PCT: procalcitonin; CRP: C-reactive protein; WBC: white blood cell; PSI: Pneumonia Severity Index; CURB-65: confusion, urea $>7 \mathrm{mmol} \cdot \mathrm{L}^{-1}$, respiratory rate $\geqslant 30$ breaths $\cdot \mathrm{min}^{-1}$, blood pressure $<90 \mathrm{mmHg}$ (systolic) or $\leqslant 60 \mathrm{mmHg}$ (diastolic), age $\geqslant 65$ years. Data on emergency room presentation are shown. Area under the curve: FGF21, 0.73; PCT, 0.62; CRP, 0.47; WBC count, 0.31; PSI, 0.76; CURB-65, 0.60 (table 4).

\section{Temporal dynamics of FGF21 and mortality}

We calculated the change in FGF21 levels from day 1 to day $3(\Delta \mathrm{FGF} 21)$ in the patient cohort from the STEP trial. Nonsurviving patients were prone to have an increase in FGF21 levels when compared with survivors $(\mathrm{p}=0.06)$ (figure $4 \mathrm{a})$. When considering only patients randomised to placebo, $\Delta$ FGF21 was significantly higher in nonsurviving patients, while it remained stable in survivors $(\mathrm{p}=0.002)$ (figure $4 \mathrm{c}$ ).

\section{Discussion}

The key findings of this study involving patients with moderate-to-severe CAP from two randomised controlled trials (RCTs) are 3-fold. First, serum levels of FGF21 measured on emergency presentation were markedly increased in the context of systemic inflammation, when compared with healthy volunteers and reference populations [10, 27]. Second, FGF21 significantly correlated with severity and outcome of CAP, yielding a prognostic accuracy as high as the PSI and higher than inflammatory parameters commonly

\section{TABLE 4 Prediction of 30-day mortality: area under the receiver operating characteristic curve} plot (AUC) analysis

$\operatorname{AUC}(95 \% \mathrm{CI})$

p-value

\section{Biomarkers}

FGF21

C-reactive protein

Procalcitonin

$0.47(0.37-0.58)$

$0.62(0.53-0.71)$

$<0.001$

White blood cell count

$0.31(0.22-0.39)$

0.03

Established scoring systems

Pneumonia Severity Index

$0.76(0.66-0.81)$

0.43

CURB-65

$0.60(0.50-0.70)$

0.01

FGF21: fibroblast growth factor 21; CURB-65: confusion, urea $>7 \mathrm{mmol} \cdot \mathrm{L}^{-1}$, respiratory rate $\geqslant 30$ breaths $\cdot \mathrm{min}^{-1}$, blood pressure $<90 \mathrm{mmHg}$ (systolic) or $\leqslant 60 \mathrm{mmHg}$ (diastolic), age $\geqslant 65$ years. ${ }^{\#}$ : versus FGF21. 
TABLE 5 Association of baseline fibroblast growth factor 21 levels with demographic characteristics, comorbidities and clinical variables

\begin{tabular}{|c|c|c|c|c|}
\hline & \multicolumn{2}{|l|}{ Univariate model } & \multicolumn{2}{|l|}{ Multivariate model $^{\#}$} \\
\hline \multicolumn{5}{|l|}{ General characteristics } \\
\hline Age & $8.7\left(\begin{array}{ll}4.9 & 12.4\end{array}\right)$ & $<0.001$ & $-1.5(-7.1-4.1)$ & 0.59 \\
\hline Male & $-171.7(-310.0--33.3)$ & 0.02 & $-238.4(-396.4--80.3)$ & 0.003 \\
\hline $\mathrm{BMI}$ & $-5.0(-19.1-9.0)$ & 0.48 & $-5.7(-19.5-8.1)$ & 0.42 \\
\hline Smoking status & $-76.5(-259.9-106.9)$ & 0.41 & $69.8(-120.9-260.4)$ & 0.47 \\
\hline COPD & $128.4(-57.1-313.8)$ & 0.18 & $-10.1(-199.9-179.8)$ & 0.92 \\
\hline Asthma & $-151.6(-541.0-237.8)$ & 0.45 & $3.1(-374.5-380.7)$ & 0.99 \\
\hline Heart failure & $477.8(290.4-665.1)$ & $<0.001$ & $226.9(13.7-440.1)$ & 0.04 \\
\hline Cerebrovascular disease & $68.5(-213.9-350.9)$ & 0.63 & $-35.7(-317.5-246.1)$ & 0.80 \\
\hline Renal insufficiency & $562.7(416.0-709.3)$ & $<0.001$ & $255.2(72.0-438.4)$ & 0.006 \\
\hline Neoplastic disease & $148.4(-134.3-431.1)$ & 0.30 & $128.6(-173.9-431.0)$ & 0.40 \\
\hline Antibiotic pre-treatment & $-188.3(-369.9--6.8)$ & 0.04 & $-92.5(-276.2-91.3)$ & 0.32 \\
\hline
\end{tabular}

BMI: body mass index; COPD: chronic obstructive pulmonary disease. \#: multivariate model: adjusted for patient age, sex, Pneumonia Severity Index, antibiotic pre-treatment and comorbidities (diabetes mellitus, congestive heart failure, chronic renal insufficiency).

measured in clinical practice. Third, adjunct treatment with corticosteroids, which potently improved hazards for achieving clinical stability, led to significant declines of FGF21 compared with placebo.

Profound elevations of FGF21 at admission suggest that it might play a role in acute metabolic adaptations in the context of systemic inflammatory responses. In fact, compared with data from healthy volunteers, levels were manifold increased and remained elevated even after 3 days [8]. Interestingly, patients with $\mathrm{CHF}$ and CKD were found to have more pronounced increases in FGF21. This is in line with previous data showing that levels of FGF21 are progressively increased from early to end stages of CKD [28, 29].

So far, FGF21 has been considered as being a starvation-induced metabolic hormone inducing genes implicated in ketogenesis and $\beta$-oxidation in mice [30]. However, in humans its role is less well characterised. It has been shown to increase only after prolonged fasting of at least 7 days duration [31], making it unlikely to facilitate acute physiological adaptations to ketogenesis and $\beta$-oxidation in humans. Additionally, it has been demonstrated that FGF21 is increased in response to insulin, such as after an oral glucose tolerance test [32] or in states of insulin resistance [33]. However, when compared with chronic metabolic conditions, levels of FGF21 were considerably higher in the majority of included CAP patients. In fact, recent studies have demonstrated that FGF21 was notably elevated in states of systemic inflammation such as systemic inflammatory response syndrome and sepsis, both in rodents [19] and humans [27, 34]. Our results describe for the first time an association of FGF21 with disease severity and outcome in CAP. FGF21 significantly correlated with disease severity (i.e. PSI), and was strongly associated with prolonged TTCS, antibiotic treatment and length of hospital stay. In line with a role for FGF21 in sepsis, its levels correlated relatively well with PCT, which is a well-established biomarker for bacterial infection and sepsis [35]. This notion is supported by animal data demonstrating release of FGF21 into the circulation in response to administration of bacterial lipopolysaccharides. Due to limited data on the causative pathogens of pneumonia in the STEP and ProCAP trials we could not establish whether FGF21 is especially elevated in bacterial versus viral infections of the lower respiratory tract system.

Moreover, it seems unlikely that the rise in FGF21 in our patient cohorts was due to reduced food intake in response to acute illness. It has been suggested that the rise of FGF21 in critically ill patients results from strongly upregulated hepatic FGF21 expression (as observed in animal models), possibly driven at least in part by mitochondrial damage [27]. Indeed, FGF21 has been demonstrated to be a sensitive biomarker of mitochondrial dysfunction [36]. Another factor to be taken into account is the relative insulin resistance caused by acute illness. Indeed, a further analysis of the FGF21 levels in the STEP trial showed a weak albeit statistically significant association of FGF21 levels with plasma glucose levels on admission $\left(\mathrm{R}^{2}=0.025 ; \mathrm{p}<0.001\right)$ (supplementary figure $\mathrm{S} 8$ ). However, given the moderate degree of chronic 

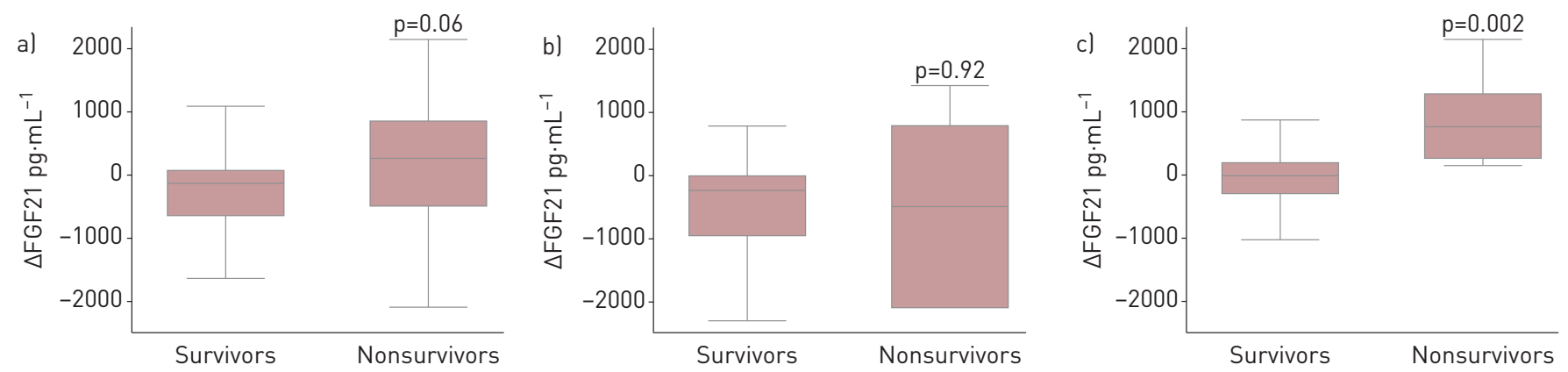

FIGURE 4 Box plots for change from baseline in fibroblast growth factor 21 ( $\Delta$ FGF21) at day 3 stratified by survivors versus nonsurvivors for a) all patients, b) patients randomised to prednisone and c) patients randomised to placebo. Nonsurvivors were prone to have an increase in FGF21 levels between day 1 and day 3 .

FGF21 elevation seen in diabetic patients, i.e. levels which are merely $\sim 2$-fold higher than in healthy subjects [33], this does not fully explain the much higher values seen in CAP patients.

Remarkably, we found that upon admission to the emergency department, patients with FGF21 levels in the highest tertile were at $>3$-fold increased risk of 30-day mortality, indicating that FGF21 could serve as a novel biomarker for outcome prediction. FGF21 was found to perform better than established routine inflammatory markers (i.e. CRP and PCT) for the identification of patients at risk for fatal outcome. Even after multivariable adjustment, FGF21 levels were still independently predictive of adverse clinical outcome.

FGF21 levels were also compared with commonly used clinical scoring systems. According to our data, the simple measurement of FGF21 provided prognostic information equivalent to the complex 20-variable PSI [37]. However, the PSI clearly performed very well in terms of mortality prediction: only one patient who was misclassified into the low-risk categories I-III died within 30 days. Nevertheless, a major limitation for the routine use of the PSI score is its laborious calculation. In a study validating the predictive potential of various indices in 731 patients with CAP, the PSI score could be calculated in only $70 \%$ of all patients [38], restricting its widespread adoption. Moreover, data from Australia have shown that the PSI is routinely used only in 6-27\% of cases. Even emergency and respiratory physicians only infrequently used the PSI and were unable to apply it accurately [7]. We show that FGF21 performed similarly to the PSI in predicting 30-day mortality using ROC analysis. Only two of the nonsurvivors had an admission FGF21 level which was not above the 95th percentile of the healthy elderly reference population. Hence, measurement of FGF21, ideally at the point of care, with a rapid immunoassay could facilitate the fast and correct triage of patients with CAP. Additionally, besides the prognostic value of admission levels of FGF21, our data show that the temporal dynamics of FGF21 during the first 3 days of CAP were of predictive value, since rising levels were associated with adverse outcome. Against this background, repeated measurements of FGF21 could possibly further facilitate appropriate patient management. However, given the relatively low mortality in our patient populations, larger cohorts will be needed to determine whether FGF21 is superior to the PSI in determining 30-day mortality or vice versa.

Since experimental data suggest that treatment with exogenous FGF21 may be protective in endotoxaemia by reducing the mortality, the observed increase in circulating FGF21 during systemic inflammation might even be a protective counter-regulatory response [19]. Thus, in addition to the diagnostic value of FGF21, it might also be a potential treatment option in severe pneumonia with sepsis. Clinical trials in humans are needed to investigate possible therapeutic applications of FGF21 in critical illness.

In animal studies, glucocorticoids have been shown to induce FGF21 in a feed-forward loop. In mice, dexamethasone induced FGF21 expression in the liver by acting on the glucocorticoid receptor. The transcription of FGF21 was co-stimulated by peroxisome proliferator-activated receptor- $\alpha$; FGF21, in turn, enhanced gene expression in the adrenal gland via the central nervous system, which resulted in an increase of corticosterone [39]. Since patients from the STEP trial were randomised to treatment with $50 \mathrm{mg}$ prednisone daily versus placebo, we investigated the effects of the corticosteroid treatment on FGF21 levels after 3 days. In contrast to the animal data, adjunct treatment with prednisone, which was associated with a shorter TTCS, reduced FGF21 levels compared with placebo. As patients on immunosuppressive medication were by protocol excluded from the STEP trial, the patient's medication seems unlikely as a possible systemic confounder that could have had an immunomodulatory effect. However, one patient who was incorrectly classified as low risk by FGF21 levels from the ProCAP trial was on glucocorticoids. This might have reduced FGF21 levels and is a potential caveat when considering the use of FGF21 as a prognostic marker. 
Our study has the following limitations. First, the results apply only to patients with more severe pneumonia, who require admission to the emergency department and, in most cases, hospitalisation. Second, we performed a secondary analysis of two RCTs which were not originally designed to explore the current hypothesis. Third, the outcome of pneumonia was significantly better in the more recent STEP trial than in the ProCAP trial. This seems to be primarily due to advances in the management of severe pneumonia over recent years. The main strengths of our study include the validation of the findings in two independent large RCTs with well-defined patient cohorts, the assessment of clinically relevant end-points, and the use of robust and precise measurement methods.

In conclusion, in two independent large RCTs involving patients with CAP, FGF21 strongly correlated with disease severity and was identified as an early predictor of adverse outcome. Further studies are required to characterise the role of FGF21 in the regulation of immunometabolism during the progression and remission of septic conditions, and to elucidate whether targeting this pathway would be of therapeutic benefit.

Acknowledgements: We thank the members of the ProCAP and STEP study teams for patient recruitment and data organisation.

Author contributions: C. Wolffenbuttel, F. Ebrahimi, M. Christ-Crain and M.J. Betz were involved in the conception, hypothesis delineation, design of the study and interpretation of data. F. Ebrahimi and M.J. Betz performed the measurements of FGF21. B. Mueller, C.A. Blum, P. Schuetz, C. Meier and M. Kraenzlin contributed data and were involved in revising the manuscript critically. All authors approved the final version to be published. F. Ebrahimi and M.J. Betz had full access to all of the data in the study and take responsibility for the integrity of the data and the accuracy of the data analysis.

Conflict of interest: None declared.

Support statement: This study was supported by grants from the Swiss National Science Foundation (SNSF PP0P3_123346) and by the Nora van Meeuwen Häfliger Stiftung and the Gottfried \& Julia Bangerter-Rhyner Foundation to M. Christ-Crain. F. Ebrahimi was supported by the Swiss Academy of Medical Sciences and the Gottfried \& Julia Bangerter-Rhyner Foundation, and by Research Funds of the University of Basel. M.J. Betz was supported by a grant from the Goldschmidt-Jacobson Foundation, Basel. Funding information for this article has been deposited with the Crossref Funder Registry.

\section{References}

1 Thomas CP, Ryan M, Chapman JD, et al. Incidence and cost of pneumonia in Medicare beneficiaries. Chest 2012; 142: 973-981.

2 Prina E, Ranzani OT, Torres A. Community-acquired pneumonia. Lancet 2015; 386: 1097-1108.

3 World Health Organization. Fact sheet: the top 10 causes of death. 2018. www.who.int/news-room/fact-sheets/ detail/the-top-10-causes-of-death Date last accessed: December 11, 2018.

4 Müller B, Harbarth S, Stolz D, et al. Diagnostic and prognostic accuracy of clinical and laboratory parameters in community-acquired pneumonia. BMC Infect Dis 2007; 7: 10.

5 Angus DC, Marrie TJ, Obrosky DS, et al. Severe community-acquired pneumonia: use of intensive care services and evaluation of American and British Thoracic Society Diagnostic criteria. Am J Respir Crit Care Med 2002; 166: 717-723.

6 National Institute for Health and Care Excellence. Pneumonia in adults: diagnosis and management. 2014. www. nice.org.uk/guidance/cg191/resources/pneumonia-in-adults-diagnosis-and-management-pdf-35109868127173 Date last accessed: December 11, 2018.

7 Serisier DJ, Williams S, Bowler SD. Australasian respiratory and emergency physicians do not use the pneumonia severity index in community-acquired pneumonia. Respirology 2013; 18: 291-296.

8 Fisher FM, Maratos-Flier E. Understanding the physiology of FGF21. Annu Rev Physiol 2016; 78: 223-241.

9 Angelin B, Larsson TE, Rudling M. Circulating fibroblast growth factors as metabolic regulators - a critical appraisal. Cell Metab 2012; 16: 693-705.

10 Hanssen MJW, Broeders E, Samms RJ, et al. Serum FGF21 levels are associated with brown adipose tissue activity in humans. Sci Rep 2015; 5: 10275.

11 Lee P, Brychta RJ, Linderman J, et al. Mild cold exposure modulates fibroblast growth factor 21 (FGF21) diurnal rhythm in humans: relationship between FGF21 levels, lipolysis, and cold-induced thermogenesis. J Clin Endocrinol Metab 2013; 98: E98-102.

12 Lee P, Linderman JD, Smith S, et al. Irisin and FGF21 are cold-induced endocrine activators of brown fat function in humans. Cell Metab 2014; 19: 302-309.

13 Chau MDL, Gao J, Yang Q, et al. Fibroblast growth factor 21 regulates energy metabolism by activating the AMPK-SIRT1-PGC1alpha pathway. Proc Natl Acad Sci USA 2010; 107: 12553-12558.

14 Kim KH, Lee M-S. FGF21 as a stress hormone: the roles of FGF21 in stress adaptation and the treatment of metabolic diseases. Diabetes Metab J 2014; 38: 245-251.

$15 \mathrm{Kim} \mathrm{KH}$, Jeong YT, Oh $\mathrm{H}$, et al. Autophagy deficiency leads to protection from obesity and insulin resistance by inducing Fgf21 as a mitokine. Nat Med 2013; 19: 83-92.

16 Brealey D, Brand M, Hargreaves I, et al. Association between mitochondrial dysfunction and severity and outcome of septic shock. Lancet 2002; 360: 219-223.

17 Saberi F, Heyland D, Lam M, et al. Prevalence, incidence, and clinical resolution of insulin resistance in critically ill patients: an observational study. JPEN J Parenter Enteral Nutr 2008; 32: 227-235. 

Pharm Des 2008; 14: 1887-1899. leptin-deficient $o b / o b$ mice from the toxicity of sepsis. Endocrinology 2012; 153: 2689-2700.

20 Li J-Y, Wang N, Khoso MH, et al. FGF-21 elevated IL-10 production to correct LPS-induced inflammation. Inflammation 2018; 41: 751-759.

21 Christ-Crain M, Stolz D, Bingisser R, et al. Procalcitonin guidance of antibiotic therapy in community-acquired pneumonia: a randomized trial. Am J Respir Crit Care Med 2006; 174: 84-93.

22 Blum CA, Nigro N, Briel M, et al. Adjunct prednisone therapy for patients with community-acquired pneumonia: a multicentre, double-blind, randomised, placebo-controlled trial. Lancet 2015; 385: 1511-1518.

23 Blum CA, Nigro N, Winzeler B, et al. Corticosteroid treatment for community-acquired pneumonia - the STEP trial: study protocol for a randomized controlled trial. Trials 2014; 15: 257.

24 Mandell LA, Wunderink RG, Anzueto A, et al. Infectious Diseases Society of America/American Thoracic Society consensus guidelines on the management of community-acquired pneumonia in adults. Clin Infect Dis 2007; 44: Suppl. 2, S27-S72.

25 Fine MJ, Auble TE, Yealy DM, et al. A prediction rule to identify low-risk patients with community-acquired pneumonia. N Engl J Med 1997; 336: 243-250.

26 Zhen EY, Jin Z, Ackermann BL, et al. Circulating FGF21 proteolytic processing mediated by fibroblast activation protein. Biochem J 2016; 473: 605-614.

27 Thiessen SE, Vanhorebeek I, Derese I, et al. FGF21 response to critical illness: effect of blood glucose control and relation with cellular stress and survival. J Clin Endocrinol Metab 2015; 100: E1319-E1327.

28 Hindricks J, Ebert T, Bachmann A, et al. Serum levels of fibroblast growth factor-21 are increased in chronic and acute renal dysfunction. Clin Endocrinol 2014; 80: 918-924.

29 Lin Z, Zhou Z, Liu Y, et al. Circulating FGF21 levels are progressively increased from the early to end stages of chronic kidney diseases and are associated with renal function in Chinese. PLoS One 2011; 6: e18398.

30 Potthoff MJ, Inagaki T, Satapati S, et al. FGF21 induces PGC-1alpha and regulates carbohydrate and fatty acid metabolism during the adaptive starvation response. Proc Natl Acad Sci USA 2009; 106: 10853-10858.

31 Fazeli PK, Lun M, Kim SM, et al. FGF21 and the late adaptive response to starvation in humans. J Clin Invest 2015; 125: 4601-4611.

32 Samms RJ, Lewis JE, Norton L, et al. FGF21 is an insulin-dependent postprandial hormone in adult humans. J Clin Endocrinol Metab 2017; 102: 3806-3813.

33 Chavez AO, Molina-Carrion M, Abdul-Ghani MA, et al. Circulating fibroblast growth factor-21 is elevated in impaired glucose tolerance and type 2 diabetes and correlates with muscle and hepatic insulin resistance. Diabetes Care 2009; 32: 1542-1546.

34 Gariani K, Drifte G, Dunn-Siegrist I, et al. Increased FGF21 plasma levels in humans with sepsis and SIRS. Endocr Connect 2013; 2: 146-153.

35 Schuetz P, Wirz Y, Sager R, et al. Effect of procalcitonin-guided antibiotic treatment on mortality in acute respiratory infections: a patient level meta-analysis. Lancet Infect Dis 2018; 18: 95-107.

36 Suomalainen A, Elo JM, Pietiläinen KH, et al. FGF-21 as a biomarker for muscle-manifesting mitochondrial respiratory chain deficiencies: a diagnostic study. Lancet Neurol 2011; 10: 806-818.

37 Lim WS, van der Eerden MM, Laing R, et al. Defining community acquired pneumonia severity on presentation to hospital: an international derivation and validation study. Thorax 2003; 58: 377-382.

38 Ewig S, de Roux A, Bauer T, et al. Validation of predictive rules and indices of severity for community acquired pneumonia. Thorax 2004; 59: 421-427.

39 Patel R, Bookout AL, Magomedova L, et al. Glucocorticoids regulate the metabolic hormone FGF21 in a feed-forward loop. Mol Endocrinol 2015; 29: 213-223. 